\title{
Seed Sterilization and Seedling Growth on Plates in the Model Grass Brachypodium distachyon
}

Aylin S Haas and Michael T Raissig*

Centre for Organismal Studies Heidelberg, Heidelberg University, Heidelberg, Germany

*For correspondence: michael.raissig@cos.uni-heidelberg.de

[Abstract] Brachypodium distachyon is a model grass closely related to wheat and barley. New resources and methods are being developed and Brachypodium is becoming a popular model for developmental biology, cell wall biology, (phylo) genomics, and genome evolution (Scholthof et al., 2018). Sterilization of seeds and seedling growth on plates is the first step to start working with Brachypodium. This is a cheap and straightforward protocol that was successfully used before (Raissig et al., 2016 and 2017).

Keywords: Brachypodium, Seed sterilization, Germination, Seedling growth on plate, Grasses

\section{Materials and Reagents}

1. Square Petri dishes (Thermo fisher scientific, catalog number: 11708573)

2. Microcentrifuge tubes $(1.5 \mathrm{ml})$

3. $3 \mathrm{M}^{\mathrm{TM}}$ Micropore ${ }^{\mathrm{TM}}$ Surgical Tape Standard $\left(3 \mathrm{M}^{\mathrm{TM}}\right.$, catalog number: $\left.1530-0\right)$

4. Pipette tips

5. Brachypodium seeds

6. $70 \%$ ethanol

7. $95-100 \%$ ethanol

8. Sterile water

9. Household bleach ("regular strength" with $5.25 \%$ sodium hypochlorite)

10. Triton X-100 (e.g., Carl Roth, catalog number: 3051.2)

11. Agar (e.g., Phyto agar, Duchefa Biochemie, catalog number: P1003.1000)

12. Potassium hydroxide $(\mathrm{KOH})$ pellets (e.g., Sigma Aldrich, catalog number: 30603-1KG)

13. MS (Murashige \& Skoog, basal, without vitamins (Duchefa Biochemie, catalog number: M0221))

14. Sterilization solution (see Recipes)

15. $1 / 2$ MS Media (see Recipes)

16. $1 \mathrm{M} \mathrm{KOH}$ (see Recipes)

\section{Equipment}

1. Pipette

2. Rocker (e.g., Benchmark Scientific Roto-Bot ${ }^{\mathrm{TM}}$ ) 
3. Flame sterilized forceps with blunt end

4. $4{ }^{\circ} \mathrm{C}$ room or fridge

5. Growth chamber (e.g., Percival or Conviron)

6. Ethanol burner

\section{Procedure}

A. Seed sterilization

1. Peel off husk (lemma with awn, Figures $1 A$ and 1B) using forceps or fingers.

Note: Put forceps at awn, move forceps along the seed to detach lemma, grab lemma and pull down. This should remove the whole lemma. Be careful not to hurt the embryo, rather leave some of the lemma than cracking the seed.

A

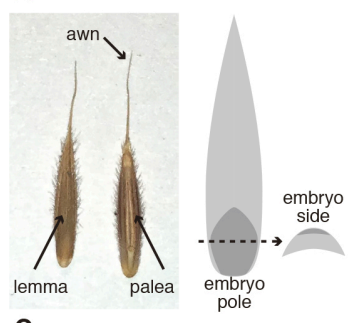

C

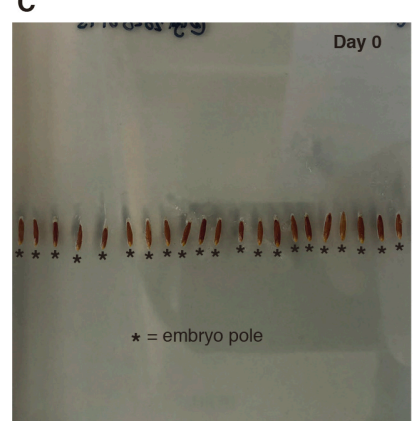

B

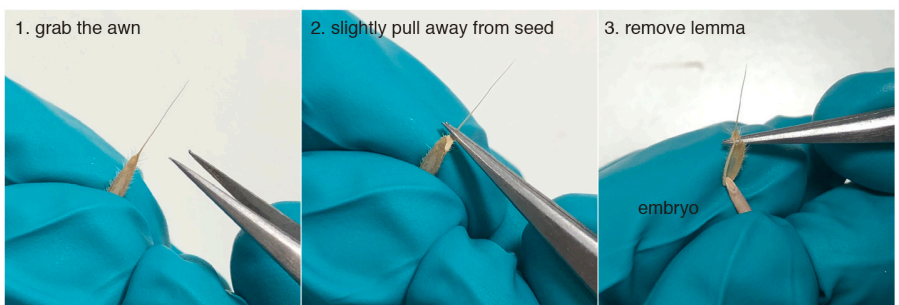

D

E

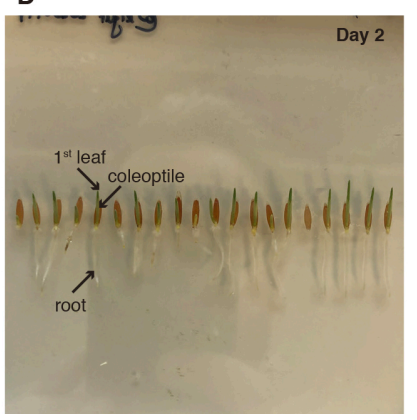

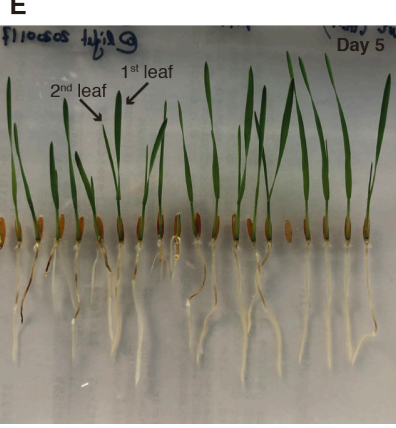

Figure 1. Brachypodium distachyon seed anatomy, seed peeling and seed germination.

A. Seed anatomy; the lemma covers the convex side and the palea covers the concave side. After removing the lemma the slightly lighter colored embryo is visible at the embryo pole (see also $1 \mathrm{~B}$, third panel). B. Steps to dehusk the seed before sterilization by peeling off the lemma. C. Plated seeds with the embryo pole pointing downwards and the convex embryo side facing up (i.e., towards the lid). D. Germinated seeds with root, coleoptile and first leaf. E. 5 days old seedlings with emerging second leaf.

2. Optional procedure for enhanced sterilization

a. In order to obtain a better sterilization also remove the palea of the seeds (Figure 1A). For this, put a maximum amount of 50 Brachypodium seeds in a microcentrifuge tube and add

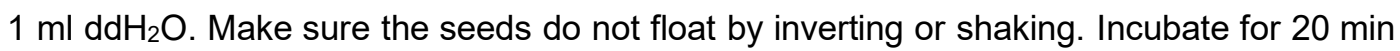
to soften the husk. 
b. Take out seeds and peel off husk (lemma with awn) and the palea using forceps.

c. Continue with Step A3.

3. Place the dehusked seeds in a clean microcentrifuge tube (maximum 50 seeds/tube).

4. Add $1 \mathrm{ml}$ of sterilization solution to the tube. Make sure the seeds do not float by inverting or shaking the tube until all seeds are immersed.

5. Incubate for $\mathbf{1 5}$ to $20 \mathrm{~min}$ on a rocker or nutator. If no such equipment is available let stand and make sure to invert tubes every 2-3 min.

6. In the meantime prepare the sterile hood. Switch on hood and clean all surfaces with $70 \%$ ethanol and bring the required equipment (pipette, sterile tips, sterile water, and vessel for liquid and solid waste). Perform the remaining steps in the sterile hood.

7. Remove sterilization solution from the tube with a pipette.

8. Add $1 \mathrm{ml}$ of sterile $\mathrm{ddH}_{2} \mathrm{O}$ and mix by inverting the tube several times to wash the seeds.

9. Remove $\mathrm{ddH}_{2} \mathrm{O}$ from the tube with a pipette.

10. Repeat Steps $A 7$ and $A 8$ two times.

11. Add $1 \mathrm{ml}$ of sterile $\mathrm{ddH}_{2} \mathrm{O}$ and place the tube at $4{ }^{\circ} \mathrm{C}$ in a dark box.

a. For two days, to synchronize germination and vernalize (cold-induced flowering).

b. For five days, for a stronger vernalization and quicker flowering.

c. Seeds can be stored in water for up to two weeks when kept in darkness and at $4{ }^{\circ} \mathrm{C}$.

Note: Vernalization can also be conducted after plating. For this, continue with B1-B6.

B. Plating

1. Sterilize forceps by dipping them in $95-100 \%$ ethanol and quickly holding them into an open flame.

2. Take seeds out of the tube using the flame-sterilized forceps.

3. Place seeds on $1 / 2 \mathrm{MS}, 1 \%$ agar plates in rows (Figure 1C). Use square plates (i.e., not round plates). Depending on the aim a plate can hold between 20 to 50 seeds. Be careful to have the embryo side of the seed facing up (convex side of the seed) and the embryo pole facing downwards (Figures $1 \mathrm{~A}$ and $1 \mathrm{C}$ ).

4. Seal plates with medical tape.

5. If not vernalized in Step A11, vernalize seeds on plates for 2 to 5 days in a dark box at $4{ }^{\circ} \mathrm{C}$ (see also Step A11 for more details).

6. Move plates to a growth chamber (16h:8h light dark cycle, $100-200 \mu \mathrm{mol} \mathrm{m} / \mathrm{s}, 20-28{ }^{\circ} \mathrm{C}$ ) and place them vertically at a $90^{\circ}$ angle. Either lean plates against the wall (several plates can be placed back to back without affecting germination or growth) or use a custom-made vertical Petri dish holder (e.g., Thingiverse, thing: 2743521)

a. Roots and coleoptile emerge after 1-2 days (Figure 1D).

b. $2^{\text {nd }}$ leaf emerges after 4-6 days (depending on temperature; Figure 1E). 


\section{Recipes}

1. Sterilization solution

$20 \%$ household bleach ("regular strength" with $5.25 \%$ sodium hypochlorite)

$0.1 \%$ Triton X-100

In sterile water, store at room temperature

2. $1 / 2 \mathrm{MS}$ Media $(1 \mathrm{~L})$

$4.3 \mathrm{~g} \mathrm{MS}$ (Murashige \& Skoog, basal, without vitamins)

$10 \mathrm{~g}$ Agar

Dissolve in $1 \mathrm{LddH} \mathrm{H}_{2} \mathrm{O}$

Adjust $\mathrm{pH}$ to 5.7 with $1 \mathrm{M} \mathrm{KOH}$

Store at $4{ }^{\circ} \mathrm{C}$ flat and agar side up

3. $1 \mathrm{M} \mathrm{KOH}(1 \mathrm{~L})$

$56.11 \mathrm{~g} \mathrm{KOH}$ in $1 \mathrm{~L}$ of $\mathrm{ddH}_{2} \mathrm{O}$

\section{Acknowledgments}

This work was supported by the Emmy Noether Programme grant RA 3117/1-1 (to MTR). This protocol was previously established (e.g., Sauer and Burroughs, 1986) and was adapted by Emily Abrash, Michael T Raissig and Dominique C Bergmann to be used with Brachypodium seeds.

Competing interests: The authors declare no conflicts of interest.

\section{References}

1. Raissig, M. T., Abrash, E., Bettadapur, A., Vogel, J. P. and Bergmann, D. C. (2016). Grasses use an alternatively wired bHLH transcription factor network to establish stomatal identity. Proc Natl Acad Sci U S A 113(29): 8326-8331.

2. Raissig, M. T., Matos, J. L., Anleu Gil, M. X., Kornfeld, A., Bettadapur, A., Abrash, E., Allison, H. R., Badgley, G., Vogel, J. P., Berry, J. A. and Bergmann, D. C. (2017). Mobile MUTE specifies subsidiary cells to build physiologically improved grass stomata. Science 355(6330): 1215-1218.

3. Sauer, D. B. and Burroughs, R. (1986). Disinfection of seed surfaces with sodium hypochlorite. Phytopathology 76: 745-749.

4. Scholthof, K. B. G., Irigoyen, S., Catalan, P. and Mandadi, K. K. (2018). Brachypodium: a monocot grass model genus for plant biology. Plant Cell 30(8): 1673-1694. 